\title{
Reclamation's Empirical Method for Estimating Life Loss Due to Dam Failure
}

\author{
Bruce Feinberg ${ }^{1}$, William Engemoen ${ }^{2}$, William Fiedler ${ }^{3}$, Dan Osmun ${ }^{4}$ \\ ${ }^{1}$ Hydraulic Engineer, Bureau of Reclamation, Flood Hydrology and Consequences Group, Denver CO, bfeinberg@usbr.gov \\ ${ }^{2}$ Civil Engineer, Bureau of Reclamation, Geotechnical Services Division, Denver CO, wengemoen@usbr.gov \\ ${ }^{3}$ Civil Engineer, Bureau of Reclamation, Geotechnical Services Division, Denver CO, wfiedler@usbr.gov \\ ${ }^{4}$ Civil Engineer, Bureau of Reclamation, Geotechnical Services Division, Denver, CO dosmun@usbr.gov
}

\begin{abstract}
The Bureau of Reclamation (Reclamation) estimates life loss resulting from dam failure in support of dam safety risk analysis. To estimate life loss, Reclamation relies primarily on an empirical methodology which is tied to the interpretation of case histories of dam failures and other types of flood disaster events. The method is known as Reclamation's Consequences Estimation Methodology, or RCEM. RCEM is a revision to an empirical method used by Reclamation since 1999, for which the case history database has been substantially expanded. Case histories, which come from dam failures, flash flood and coastal flooding events, are the basis for data points used in the estimation of fatality rates. Reclamation's RCEM methodology employs a graphical approach for the selection of fatality rates based on ranges of DV (depth multiplied by velocity) and warning time. DV is used to quantify the intensity of flooding and can be related to lethality. The RCEM methodology is in many ways similar to Reclamation's previous method, DSO-9906 . However, the new method requires greater application of judgment when selecting fatality rates. The paper will provide an overview of the new method, with emphasis on the case histories, and discussion of the importance of a team approach to life loss estimation.
\end{abstract}

\section{Introduction and Overview of RCEM 2014}

The Bureau of Reclamation uses a risk-informed approach to manage and prioritize the dams within its dam safety program. Dam failure probability and life loss consequence estimates are used to quantify risk. Reclamation's life loss estimates have been and will continue to be primarily founded in an empirical approach which makes use of dam failure and other flood related case histories as a basis for the selection of fatality rates. RCEM 2014 defines fatality rate as the total number of fatalities divided by the affected population at risk.

The previous Reclamation methodology for estimating life loss from dam failure, DSO-99-06, has been revised. Reclamation's Consequence Estimating Methodology (RCEM 2014) replaces DSO-99-06 and offers a number of improvements as a result of the lessons learned from using DSO-99-06 over a 15 year period. RCEM 2014 fatality rates are based on case history data which has been expanded by 50 percent over the original 40 dam failure and other flooding case histories from DSO-99-06. This expansion of case history data helps to strengthen the empirical relationships from which fatality rate estimates are derived, and helps provide a better basis for fatality rate selection. A detailed case history document has been developed which contains descriptions and data for all the case histories used to formulate the method. With RCEM 2014, the selection of fatality rates is made from a graphical plot of case histories for which envelope curves have been developed to define a suggested range and upper and lower boundaries. The RCEM 2014 fatality rate curves are estimated based on ranges of warning time and DV which is the product of maximum flood depth and velocity. DV can be related to lethality through case histories, and the DV parameter is recognized through numerous studies as a lethality indicator.

Users of the RCEM 2014 method are encouraged to develop ranges of life loss based on possible variations of key parameters and situational outcomes. A key component of RCEM 2014 is the required documentation and justification of assumptions leading to the life loss estimates. RCEM 2014 users are encouraged to "build the case" which would involve creating a detailed written description of why certain parameter values and assumptions were selected.

RCEM 2014 consists of three documents: the methodology document which details the use of RCEM 2014, a case history document which provides a basis for the empirical estimates, and a document that presents examples of use which describes applications of the method for both simple and more complex life loss estimating scenarios related to dam safety risk analysis.

\footnotetext{
a Corresponding author: bfeinberg@usbr.gov
} 


\section{The Development of RCEM 2014}

DSO-99-06 had been in use by Reclamation for 15 years, has mostly worked well, and has provided generally consistent results that make sense. The basis for the estimates is easy to understand. Experiences of applying the method to dam safety risk analysis have identified aspects of the methodology that could be improved upon. DSO99-06 is based on 40 case histories of dam failure and other types of flooding events. Some of the case histories are obscure and not well documented. There was a need to learn more about the 40 cases and to provide this data as background information to aid in the selection of fatality rates. A number of dam failure and flooding cases existed which were not included in DSO-99-06. The development of RCEM 2014 provided the opportunity to add additional case histories, and these new cases help to increase confidence in the empirical relationships used to select fatality rates.

DSO-99-06 made use of a table for the selection of fatality rates. Based on variations in flood severity (a measure of flooding intensity which is correlated to degrees of lethality), warning time, and flood severity understanding (a perception of the public's understanding of the risks), the DSO-99-06 user could select the most appropriate choice from 15 suggested ranges of fatality rates. One limitation however, is that of these 15 fatality rate ranges, only 6 were based on data from two or more case histories, 4 were based on only one case and 5 had no direct case history and were based strictly on judgment. While the application of judgment is considered to be a viable method for creating a reasonable argument, one of the goals of RCEM 2014 was to create a more defensible method of selecting fatality rates by strengthening the association with case history data.

The DSO-99-06 method used three categories of flood severity, high, medium, and low, and the assessment of these categories was often qualitative. The method did not provide guidance for estimating fatality rates for high severity flooding where there was adequate warning. In fact, DSO-99-06 somewhat discouraged users from assuming high severity flooding. Given the large size of many Reclamation dams and reservoirs, there are numerous cases where a dam failure could lead to high severity flooding downstream.
Another concern with DSO-99-06 fatality rate selection had to do with low intensity (low severity flooding) which might occur at communities very far downstream from a breached dam, and with many, many hours or even days of warning. For this situation, there is an opinion that the DSO-99-06 fatality rate tables do not allow a range of fatality rates that is low enough to reflect the effects of this extensive amount of warning.

\section{The Graphical Approach to Fatality Rate Estimation}

With RCEM 2014, the selection of fatality rates is based on estimates of warning time and DV. Fatality rates are selected from a set of graphs as shown in Figures 1 and 2. The difference between the two graphs is the warning category. Each graph, which is a log-log plot, has DV on the $\mathrm{x}$ axis and fatality rate on the $\mathrm{y}$ axis.

The method makes use of two warning categories, little to no warning and adequate warning. These categories are not rigidly defined and it is recognized that what constitutes adequate warning can be site specific. For example, a small community directly below a dam may only need 15 minutes warning to be considered adequate, but a large urban area might need 6 hours due to traffic congestion. In this situation, anything less than 6 hours may be considered little to no warning.

Based on an estimate of DV, a range of fatality rates can be evaluated. The graphs contain two sets of curves, a suggested range and an upper/lower (envelope) bound. RCEM 2014 encourages the development of a range of fatality rate estimates for a given DV value. The suggested fatality rate range can span up to two orders of magnitude for a single DV value, so judgment must be applied to select an appropriate range of fatality rates. Examples of factors which could affect the selection of a fatality rate range may include: day vs. night, location of the affected PAR relative to the most severe portions of flooding and the corresponding differences in DV, fast rising flooding vs. slowly rising, barely adequate warning vs. many hours of advance warning. 


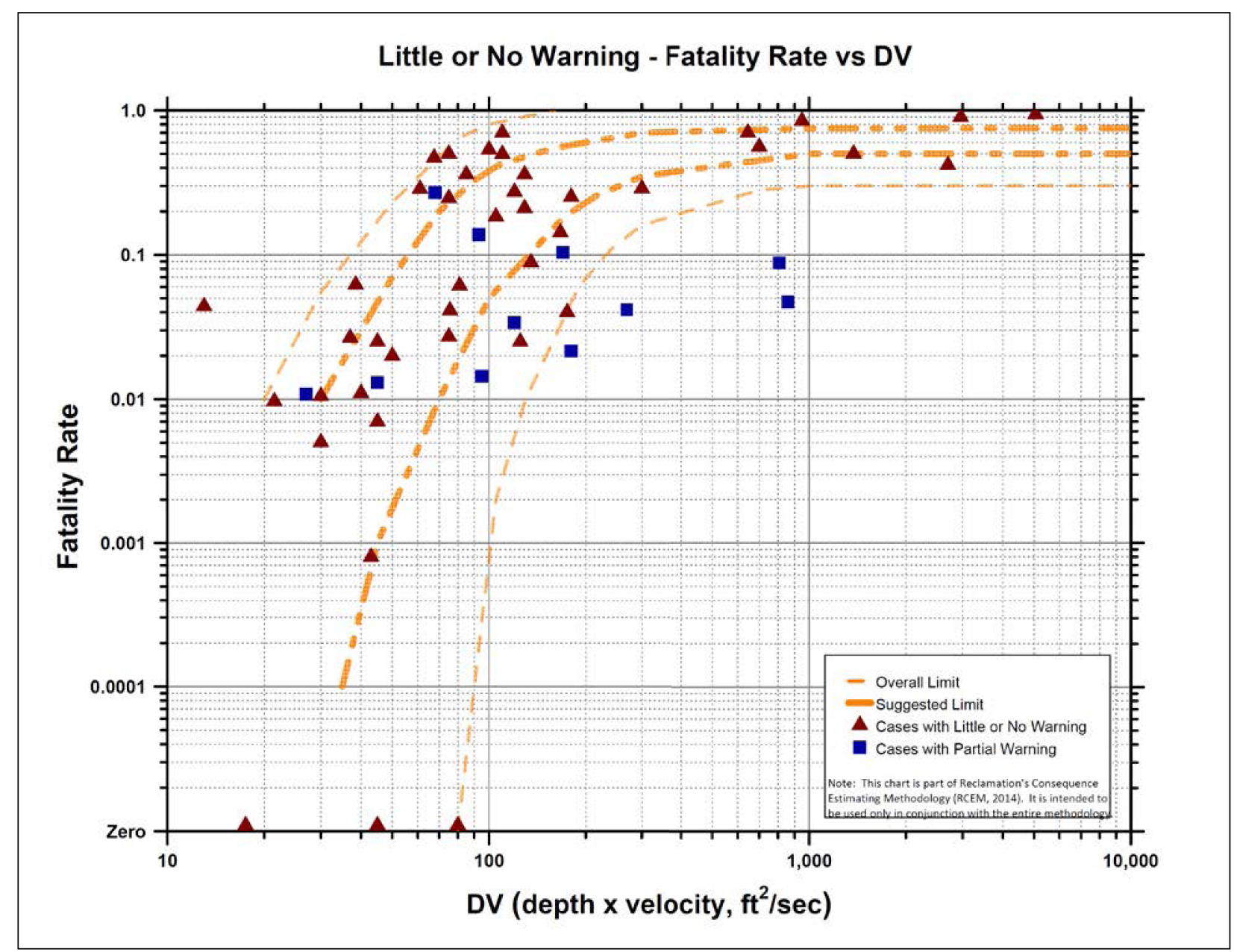

Figure 1. RCEM 2014 Fatality Rate Chart, Little to no warning 


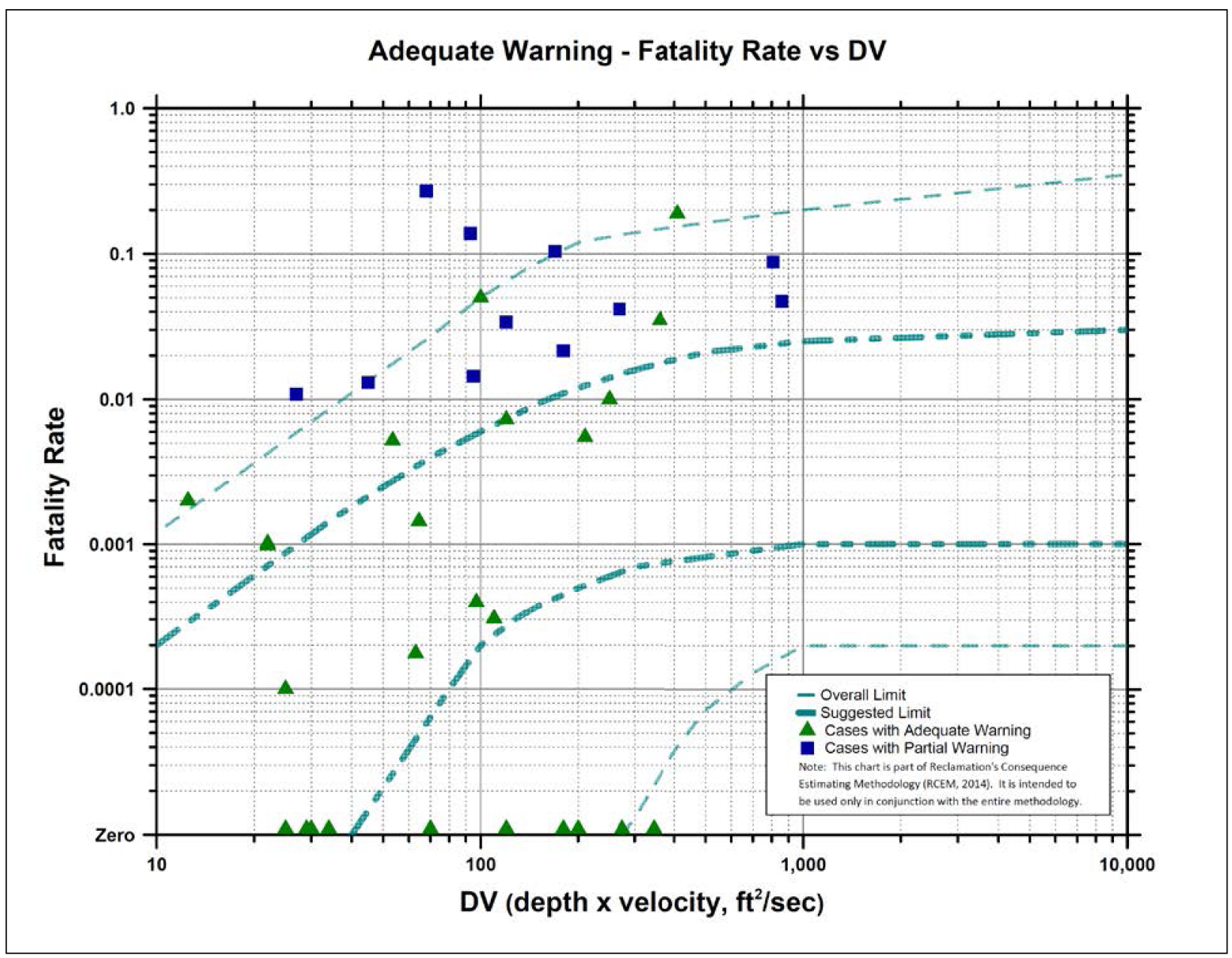

Figure 2. RCEM 2014 Fatality Rate Chart, Adequate warning

\section{Case Histories and Important Parameters}

The case history document provides a basis for the empirical relationships developed for RCEM 2014. The document provides a summary of each flood event and a table which contains relevant parameters.

The 40 case histories from DSO-99-06 are described in the document. RCEM 2014 includes an additional 20 cases. Some of the 60 cases did not contain all of the information required to be used as data points. The creation of a data point requires case history information regarding the amount of warning time, the maximum DV at the location where fatalities occurred, the affected population at risk (PAR) and the fatality rate. A total of 80 data points were developed, with some case histories containing multiple points where there was information available about different flooded reaches and variation existed regarding warning time, DV and/or fatality rate. The 80 case history data points fall into the following categories:

- Flash flood - 8 data points

- Flash flood involving dam releases - 1

- Landslide induced reservoir wave - 1

- Hydrologic overtopping - 19

- Overtopping due to mis-operation - 1

- Levee breach - 3

- Regional flood - 2

- Coastal flood (tsunami and cyclone related) -4

- $\quad$ Spillway failure - 1

- Static failure, including high reservoir cases -36

- Lahar flow - 1

- Dam failure, unknown cause - 3

Other relevant data which is compiled for each case history is presented in the case history document in a tabular format. This data, where applicable and 
available, provides information to the RCEM 2014 user when trying to make a comparison for a hypothetical dam failure case to the various case histories. The tabular data items are listed as follows:

- Flood Severity Rating - high, medium, low. RCEM 2014 quantifies flooding intensity in terms of DV and does not directly use the DSO-99-06 flood severity categories, However, the qualitative aspects of the DSO-99-06 flood severity rating can be useful when examining case histories and trying to estimate DV.

- Warning Time - little to no warning or adequate warning

- $\quad$ Time of day - fatality rates are typically higher at night

- Failure scenario - for dam failures, this parameter would describe the failure mode (e.g. flood overtopping, static internal erosion failure, etc.)

- Total (affected) population at risk (PAR)

- Number of fatalities

- Fatality Rate - number of fatalities divided by total affected PAR. Note that this differs from a mortality rate, which has been used by others to describe the total number of fatalities divided by the total number of post-evacuation PAR. The case history data includes very little information on evacuation rates. For this reason, the fatality rate is considered to be more reliable.

- Dam height - if available

- Reservoir storage - if available

- Breach formation time - if available

- Downstream Distance to PAR

- Maximum DV - estimated by a variety of methods. See the DV and Flood Severity section.

- Flood severity understanding - This is an assessment of how the downstream public perceived the risks posed by flooding. DSO99-06 made use of this parameter, but it is not explicitly included in RCEM 2014. Flood severity understanding is a very subjective parameter without much supporting data.

- Confidence in data - an assessment based on impressions of data quality and quantity and how the life loss estimates affect the total risk estimate.

The expansion of the case history data set is an improvement over the original 40 case histories used for DSO-99-06. However, it is recognized that this data set is still limited. Reclamation considers this dataset to be continuously evolving and hopes to expand the case history data in the future as new data becomes available.

\section{DV and Flood Severity}

DV has been used as a measure of the level of both destructive potential and lethality. Case history data supports the concept that higher DV can be related to higher lethality. A number of studies have shown that high DV flow affects building structural stability, stability of motor vehicles and the stability of persons caught out of doors during flooding.

DV for the RCEM 2014 case histories was estimated as a range whenever possible, and adjusted as necessary, to account for variations in location of the affected PAR relative to the most severe portions of flooding.

The DV was estimated for the RCEM 2014 case histories in various ways:

- Historic accounts of maximum depth were multiplied by velocities obtained from reported flood wave travel times. This information is often anecdotal though, and was sometimes reported by observers who may have been in a state of confusion at the time of observation. DV can also be approximated by dividing estimates of maximum discharge by the width of flooding at the same location. Care needs to be taken when using anecdotal information to make sure the results seem reasonable.

- Cross referencing between the qualitative and quantitative (numeric for low and medium severity) descriptions of flood severity from DSO-99-06 and more recently developed numeric estimation of high severity flooding in use by Reclamation. Often this approach involved making comparisons to historic photographs showing the aftermath of flooding. For example, DSO-99-06 medium severity has been described as destructive flooding where homes are destroyed but trees or mangled homes remain for people to seek refuge in or on. Medium severity flooding is also described by DSO-99-06 as having a DV of at least $50 \mathrm{ft}^{2} / \mathrm{s}$. An examination of downstream damages, through photographs, can provide information that allows the relation of a descriptive assessment to numeric values.

- Post flood surveys and investigations, such as those performed in the past by the United 
States Geologic Survey (USGS), have provided estimates of DV at downstream locations.

- DV has also been estimated from the hydraulic re-creation of numerous dam failure floods. This method is considered to be fairly reliable, and often there is some calibration which helps to increase confidence in the DV estimates.

\section{Application of the Methodology and Building the Case}

The 10 tasks performed for estimating dam failure life loss using RCEM 2014 are described as follows:

Task 1 - Select dam failure scenarios (e.g. sunny day, flood, etc.) that correspond to dam potential failure modes

The loss of life caused by dam failure flooding may be highly dependent on the potential failure mode, which includes consideration of any loading being applied to the structure and the response of the structure to the loading. Failure scenarios for dam safety risk analysis are typically identified from the findings of a Potential Failure Mode Analysis. For the purposes of dam safety risk analyses, potential failure modes usually fall into one of three categories: static, seismic, and hydrologic. While there may be a significant range of dam failure scenarios, it is not necessary to estimate life loss for every scenario; similar dam failure scenarios can be grouped together and the estimated life loss range can capture some of the variability in the dam failure scenarios.

Different potential failure modes may have similar, or widely varying, breach outflows. In addition, the speed with which the breach develops can impact many key life loss estimating factors such as warning time, size of inundation area, and flood severity.

Task 2 - Select appropriate time categories (e.g. day/night, seasonal, weekend/weekday)

The first step in this task is to evaluate if various time categories are needed to estimate life loss. In general, different time categories may be needed if the PAR varies significantly over time. If there is no significant variation in PAR over time and there are very long warning times for downstream populations, then a judgment can be made that there would not be a significant difference between day and night conditions. In this situation, only one time category is used for the life loss estimate.

The time of day, day of week, and month or season during which the dam failure takes place may strongly influence the resulting loss of life. Case histories of dam failure flooding events have shown that warning and response can be much weaker during nighttime hours, resulting in significantly higher fatality rates. The time of day can have a significant influence on life loss for situations where the PAR is very close to the dam and less of an influence where the PAR is many hours downstream. Consideration of different time categories can help with sensitivity analyses and can help estimate ranges of PAR and life loss.

PAR is typically considered to consist of permanent residents and transient population such as recreationists. Transient PAR is usually assumed to be much more variable than residential PAR; for example the transient population may decline greatly in winter when recreation opportunities along a river may be limited.

Task 3 - Review and evaluate flood inundation mapping and define appropriate reaches or areas flooded (by river reach, town, etc.) for each dam failure scenario

Flood inundation modeling is a critical part of the life loss estimation process. The flood inundation model provides estimates of the inundation areas, the severity of flooding, and flood wave travel times. It requires assumptions about the type of breach that will occur.

Flooded areas downstream from the dam can be divided into several different locations or river reaches. When deciding how to divide the inundation area, the following factors should be considered:

- Residential versus transient PAR;

- Occupancy type (e.g., tent in a campground versus one-story dwelling);

- Varying occupancy considering season, time of day, or other factors (e.g., manufacturing facilities, summer resort areas, campgrounds, picnic areas);

- Population density (e.g., scattered residences, small town, large city);

- Flood characteristics (i.e., flood depths, DV, rate of rise);

- Warning characteristics (i.e., timing, amount, and quality).

Areas with similar characteristics should typically be combined into a single reach.

Task 4 - Estimate the flood severity range (i.e., DV range) for the flooded areas

Flood severity has a significant influence on fatality rate. In general, case history data indicates that the highest estimated fatality rates are associated with the highest estimated DV values. When the flood severity is lower, there is greater observed scatter in 
the fatality rates, most likely because other factors have a greater influence at the lower DV values. Flood severity is quantified in terms of depth multiplied by velocity of flow, or DV. Although the parameter DV is not necessarily representative of the depth and velocity at any particular structure, it is representative of the general level of destructiveness that would be caused by the flooding. DV increases as peak discharge from dam failure increases, or it may decrease as the width of the inundated area increases.

Most commonly, DV can be quantitatively estimated at any location by dividing the flood flow $\left(\mathrm{ft}^{3} / \mathrm{s}\right)$ by the flood width (feet), or by multiplying maximum flood depth and maximum velocity as obtained from hydraulic modeling output information. However, there are a number of ways that DV has been, and can be, estimated depending on the availability of flood information. Since RCEM features the use of a $\log$ scale for the data, relatively small ranges in the DV parameter (perhaps factors of 2 or 3 , for example) may not significantly impact the fatality rate, depending on the location in the curve where the values fall. Although it is important to estimate DV as carefully as possible, it is not critical that the resulting calculation is completely "accurate." Rather, a range of DV can be estimated using different approaches and with varying input assumptions. In fact, the actual DV values in a given flood reach probably do vary appreciably, so providing a range may be the best way to represent conditions. In most cases, this range can be used with the graphs to come up with a reasonable fatality rate range.

Task 5 - Estimate the population at risk within each reach for each failure scenario, flood severity range, and time category

After DV values have been estimated in each flooded area, the PAR in each area is estimated. For each combination of failure scenario, DV range, and time category identified in Tasks 1, 2, and 4, the number of people at risk is estimated. PAR is defined as the number of people occupying the dam failure flood plain prior to the issuance of any warning or evacuation.

At a very basic level, the development of a PAR estimate can be as simple as visiting the area downstream of a dam and counting houses in the inundation zone. PAR can also be obtained using the inundation mapping data overlain with census data. A geographic information system (GIS) is a powerful tool that can be used to simplify this process. The most accurate data for residential PAR estimation is at the level of the census block. When the flood inundation boundary can be overlain with the census block data in a GIS, the number of inundated PAR households can be calculated. Partially inundated census blocks must be treated separately. If the residences are evenly distributed within the partially inundated block, a percent inundated estimate can be applied to the total number of households within that block. If the distribution of residences within a partially inundated block is more concentrated in specific locations, then the recommended approach would be to manually count the houses (identified in aerial imagery) in the inundation zone. Finally, the total number of inundated residences is multiplied by an average household size that is specific to the area of interest (which can be obtained from census data), to obtain the estimated residential PAR.

\section{Task 6 - Estimate when dam failure warnings would be initiated and estimate the warning time categories for flooded areas (e.g., little to no warning, adequate warning, or between the two)} Warnings refer to either specific notification of a developing or already in progress dam failure issued by public officials, or an informal recognition and awareness of a developing threat, perhaps passed by neighbors or simple observations of changing river conditions.

In the most ideal situation, a dam breach in progress would be detected well in advance of the beginning of catastrophic outflows, and warnings and a strong evacuation order would be issued to downstream PAR without delay, with all of the PAR moving safely out of the flood zone by the time flooding arrives downstream. Dam failure and flash flood case histories indicate the ideal situation does not always develop. The sequence of events that takes place is often a mix of physical and social phenomena combined with some element of chance or luck.

The recognition of a developing dam failure and the possible issuance of warning by officials and subsequent PAR decisions regarding evacuation are critical factors that impact the potential for life loss. However, an equally important consideration is flood wave travel time, or how long before the dam failure flooding actually reaches a given downstream reach. Flooding case histories show that, in general, the number of fatalities decreases as the distance downstream increases, but increasing distance by itself is not what decreases the life loss potential. Potential life loss decreases when the travel time begins to exceed the amount of time required to warn and evacuate the PAR. (Evacuation, or the lack thereof, is accounted for in the fatality rates (using the pre-evacuation PAR) described in Task 7.) Another result of increasing distance is the attenuation (reduction) in flow that occurs. However, 
flow depths and velocities can increase downstream if the flood plain transitions from a wider valley to a narrow canyon.

Assumptions regarding when formal or informal dam failure warnings for a particular dam would be initiated can be based on an analysis of the monitoring/detection mechanisms (including the likelihood that anyone will observe a changing condition), decision making, and notification systems or procedures for the dam. In many cases it may be appropriate to estimate reasonable best case and worst case situations to bracket the time when warnings would be initiated. This range of values can be used to estimate a range of warning times. The case history data generally indicates higher fatality rates for less warning time, and vice versa. However, because of the large number of factors that influence each case, similar fatality rates may result from different cases with different warning times. For the purpose of estimating warning time using RCEM, two warning time categories are used:

- Little to no warning (typically less than an hour)

- Adequate warning (typically more than an hour, although there could be situations with a dense population where hours of warning are not adequate)

After estimating the warning time range for each location, a judgment is made as to which warning category would best represent that location. The distinction is important because in Task 7, fatality rates are estimated using either a chart for "little to no warning" or a chart for "adequate warning." The exact determination of how many minutes or hours of warning is not as important as the general category selected. As discussed below under Task 7, the expected warning time (and quality) is a consideration (along with other factors) when selecting the upper and lower limits of the recommended and overall fatality rates, from either of the two charts.

Task 7 - For each PAR reach, use the graphical approach to estimate an appropriate fatality rate range based on flood severity, warning time and other considerations

This task involves using all of the information available for a dam failure scenario to estimate fatality rate ranges for each PAR area. For PAR areas that are assumed to receive little or no warning, Figure 1 is used, and for PAR areas that are assumed to receive adequate warning, Figure 2 is used. Each chart includes dashed lines that represent "suggested" and "overall" limits for fatality rates over the full range of DV values. The suggested limits provide a starting point for estimating the fatality rate range.
The selected fatality rate can be increased or decreased, based on all of the relevant factors for each specific PAR area. The full limit ranges shown are not intended to be used by estimators directly, but rather they are intended to help the estimator interpret the approximate data trends from the case histories. For example, the range of overall limits for little warning and a DV of $100 \mathrm{ft}^{2} / \mathrm{s}$ covers about three orders of magnitude; however, it is unlikely that the range of uncertainty in the fatality rate selected would span that full range. Typically, the selected fatality rate range would be expected to span about one order of magnitude. Judgment should be applied and a case should be built for selecting a fatality rate range that is most appropriate for the situation being evaluated. The application of judgment can include: comparison to relevant case histories, site specific topographic/geographic/demographic considerations, evaluation of relative changes in flow characteristics for a given reach when compared to an upstream reach, assumed differences in warning time between subsequent reaches, and the potential for evacuation. Judgment can also be applied between various potential failure modes in terms of differing flow characteristics and anticipated warning times. It is acceptable to use a fatality rate range with limits above or below the overall limits, as long as a case is built for the estimated range, particularly that portion of the range that is beyond the limits of the case history data.

\section{Task 8 - Estimate life loss range for each PAR reach by applying appropriate fatality rate range limits to each PAR reach}

The range of estimated life loss for each specific PAR reach (corresponding to a location, warning time, or flood severity) is determined by simply multiplying the appropriate fatality rate range limits by each PAR estimate. For each dam failure scenario, the life loss estimates from each PAR reach are summed to get the total estimated life loss range. In addition to providing the range of total fatalities, a "best estimate" should be provided. This best estimate may be the mean, or a weighted average between seasonal or day/night combinations. There is no "correct" way to determine this best estimate; it is up to the estimating team to build a case for the best estimate within the total estimated life loss range.

\section{Task 9 - Evaluate how uncertainties and variability in various parameters affect overall uncertainties in life loss estimates}

As evidenced by case histories, there can be a large range of fatality rates from dam failure flooding. This is not surprising, considering the variability in 
PAR, severity of flooding, and warning time. However, even within a given category of flood severity or warning time there can still be a wide range of fatality rates. These differences may result from having some of the PAR located near the river and some of the PAR located farther away and thus less likely to feel the brunt of the flood flows. Similarly, not all warnings are issued in the same manner, and different populations may respond quite differently to warnings.

The graphical approach features "overall" limits to observed fatality rates; these are essentially envelope curves that cover the majority of case history data points. Within the overall limits are a set of "suggested" limits. Even these suggested limits typically show significant differences between the upper and lower curve. Thus, case histories confirm the uncertainty and variability inherent in the potential loss of life due to flooding. It is important to recognize this uncertainty, and properly reflect it in final estimates by portraying life loss as a range, often with an order of magnitude difference between upper and lower bounds. In addition, the estimating team should consider what type of probability distribution should be applied to any reported range. It is not unusual for a life loss range to be reported as a uniform distribution, which implies that the life loss really could fall anywhere within the range with equal likelihood. This would result in a mean estimate in the middle of the range, reflecting a belief that the team finds no compelling reason that the life loss would be expected to fall in either the high or low end of the range.

Sometimes, the confidence in an estimate, as well as an understanding of uncertainty, can be enhanced by a simple sensitivity analysis. Instead of assuming only a point estimate for a particular parameter in a consequences analysis, a range in life loss can be calculated by assuming different values for that variable. Approaching a life loss evaluation in this manner will likely provide a better idea of the potential range of life loss to be expected, as well as improve the confidence in the estimate.

\section{Task 10 - Build the Case for the Life Loss Estimates}

Building the case for the selected life loss estimates is a key requirement. The case for the life loss estimates should address the key inputs that are included in the preparation of the loss of life estimates, including: available inundation studies and the failure scenarios and breach assumptions that define the flow characteristics, the accuracy of census or other information used to estimate the population at risk along the inundated area, the basis for assumptions of when warning would be issued, any limitations on warning effectiveness and/or evacuation of the population at risk, any unique site specific factors, and an overall rationale for the selection of fatality rates. The case for the consequences should convince the reader and ultimately the decision makers that the loss of life estimates are reasonable.

The case for the loss of life estimates should discuss the uncertainty inherent in the estimates and the confidence that the risk analysis team has in the estimates. If sensitivity studies indicate only small differences in the life loss estimates, confidence will be higher in the estimates. Even if the loss of life estimates are sensitive to the assumptions, if the overall dam safety findings are not changed based on the sensitivity studies, the overall confidence in the findings may remain moderate to high. For example, although the life loss estimates may vary by a factor or 2 or 3 depending on assumptions (indicating a lower confidence in the estimated life loss), the total annualized life loss estimate may still remain in the area indicating decreasing justification to take action.

\section{Comparisons to DSO-99-06}

Life loss estimates produced by RCEM have been found to be generally similar to DSO-99-06, with some exceptions. Before RCEM 2014 was adopted by Reclamation, trialing was performed to compare the results of RCEM 2014 to DSO-99-06. A variety of cases were selected in an attempt to get a representative sample of the dams within Reclamation's portfolio. This included both concrete and embankment dams, large and small dams and reservoirs, large and small downstream PAR, variations in downstream topography, variations in older and newer inundation studies, warning time variations, and dams with relatively high and low estimated life loss. Seven persons were tasked with completing the comparison estimates. Each estimate was performed by a single individual. Twenty dams were evaluated with a total of 40 dam failure scenarios, which included static, seismic and hydrologic failure modes. In addition, 5 dams and 9 life loss cases were evaluated by more than one estimator to examine the repeatability of RCEM 2014 results.

The outcome of the trials indicated that the results of using the two life loss estimating methods are mostly consistent, although there were some differences. For most of the seismic and some static dam failure cases, results with RCEM 2014 produced higher life loss numbers. This was due to the requirement in RCEM 2014 that DV be calculated. DSO-99-06 did not require a DV calculation and the flood severity rating was often a qualitative estimate. In addition, 
the DSO-99-06 method suggested that high severity flooding should be reserved for only the most extreme cases. Most Reclamation users of DSO-9906 applied medium flood severity and its corresponding fatality rates as an upper bound, even in cases which might produce very intense flooding. When DV is actually calculated, it can be seen that for many cases involving the rapid failure of high dams, DV is within the range of what might have been considered high severity flooding with DSO-9906 . So, the higher life loss numbers obtained from the trials using RCEM 2014 for seismic and some static failure cases seem to make sense.

RCEM 2014 produced lower fatality numbers for situations where PAR was located very far downstream and there was assumed to be many hours or even days of warning. The RCEM 2014 adequate warning fatality rate curve allows for the selection of very low fatality rates which are below the lower range of fatality rates suggested by DSO-99-06.

Estimated life loss from both DSO-99-06 and RCEM 2014 generally fall in the same range (particularly when the $\log$ scale of $\mathrm{N}$ is considered). When life loss is less than 100 , best estimates typically vary only a small amount, and no more than a factor of 3 . At higher life loss estimates, the maximum increase is a factor of 6; however, these differences appear to be justified.

Findings of the trials suggest that a team approach is best in that it allows for the consideration of a variety of viewpoints and opinions. A stronger, more defensible case can be developed when the life loss estimate is developed through collaboration amongst multiple individuals.

\section{Importance of a Team Approach to Life Loss Estimation}

Reclamation had used DSO-99-06 for 15 years prior to the development of RCEM. Originally, standard practice was for a single individual to estimate life loss consequences, and the findings would be used for risk analysis. Often times, questions would arise over whether certain factors were considered and accounted for in the life loss estimate. Life loss estimates performed by a single individual did not always inspire confidence in the final numbers. At Reclamation, the development of analysis leading to the Annualized Failure Probabilities (AFP) for a particular dam's potential failure modes, have long been derived using a team approach with group discussion and input. The AFP is the "other half" of the risk analysis findings, which along with life loss, helps to portray overall risk posed by a dam facility.
The question was asked, if development of the AFP makes use of a detailed, team approach, wouldn't doing the same for life loss estimation improve the overall process and increase confidence in the risk analysis findings?

The trialing effort which took place during the development of RCEM help to emphasize the perceived importance of a team approach to life loss estimation. The process of trialing employed single individuals to estimate life loss for a dam failure using RCEM and comparing the results to previous estimates using DSO-99-06. Also performed were "repeatability trials", where more than one person estimated life loss using RCEM for a given dam in order to check for variability that may show up in the estimates. In some cases significant differences occurred between estimators. Discussions between the developers of the RCEM method and the trialing estimators shed light on the fact that certain key considerations were sometimes overlooked by the trialing estimators. These findings helped to reinforce the idea that life loss estimates are best performed by a team of individuals, and that discussion and debate can lead to a consensus estimate with greater confidence in the final numbers.

There is a great deal of judgment involved in the development of life loss estimates. This is not only true of the RCEM methodology, but for any consequence evaluation. Given the many variables, and resulting uncertainty in life loss estimates, it is not unexpected that two different individuals may end up with differing life loss estimates for a given failure mode. To improve the quality of the life loss estimates, it is recommended that a team approach be utilized. Typically, within Reclamation, life loss estimates are developed as part of a risk analysis by the risk analysis team.

The team discussions help bring to light differing assumptions and opinions on a variety of factors such as flooding intensity, location of PAR, likelihood of warning and evacuation. This process can provide great value in considering varying viewpoints and arriving at a consensus estimate of estimated life loss (which admittedly contains significant uncertainty).

\section{Summary}

The RCEM 2014 method is an improvement over its predecessor, DSO-99-06. The two methods are generally similar in their approach, but RCEM 2014 incorporates a number of refinements. Expansion of the case history data set increases confidence in fatality rate selection. Development of the case 
history document provides better access to the empirical data and makes the basis for the method more transparent. The dam failure life loss estimation approach is simple, easy to use and can produce consistent results within a portfolio of dams.

RCEM 2014 should be used carefully. Judgment should be applied and estimates of life loss should be justified by building the case, which includes comparison to relevant case histories, if applicable. A team approach to life loss estimation is recommended in that it allows for discussion and evaluation of all influencing factors which contribute to the life loss estimate. Life loss estimates should be developed as ranges rather than single point values.

\section{References}

1. "RCEM - Reclamation Consequence Estimating Methodology - Interim Guidelines for Estimating Life Loss for Dam Safety Risk Analysis” Bureau of Reclamation, Denver, Colorado,

February 2014.

2. "RCEM - Reclamation Consequence Estimating Methodology - Interim Dam Failure and Flood Event Case History Compilation," Bureau of Reclamation, Denver, Colorado,

February 2014.

3. "RCEM - Reclamation Consequence Estimating Methodology - Interim Examples of Use," Bureau of Reclamation, Denver, Colorado, February 2014.

4. "A Procedure for Estimating Loss of Life Caused by Dam Failure," Dam Safety Office Report DSO-99-06, Bureau of Reclamation, Denver, Colorado, September 1999.

5. "Best Practices in Dam and Levee Safety Risk Analysis," Bureau of Reclamation and U.S. Army Corps of Engineers, December 2012.

6. "Interim Dam Safety Public Protection Guidelines - A Risk Framework to Support Dam

Safety Decision-Making," Bureau of Reclamation, Denver, Colorado, August 2011. 